\title{
Assessment of the transcription levels for the complement activation control system in eutopic endometrium in women with two or more consecutive miscarriages of unknown etiology
}

\author{
Przemysław Wirstlein, Mateusz Mikołajczyk, Jana Skrzypczak \\ Division of Reproduction, Department of Obstetrics and Gynecology, K. Marcinkowski University \\ of Medical Sciences, Poznań, Poland
}

\begin{abstract}
Human endometrium, deciuda and placenta have been shown to express factors that inhibit the complement activation cascade - decay-accelerating factor (DAF), membrane cofactor protein (MCP) and the C3 complement component. In the following study we have analyzed the transcripts levels for DAF, MCP and heparin-binding epidermal growth factorlike growth factor (HB-EGF), the $\mathrm{C} 3$ complement component and receptor for vascular endothelial growth factor (VEGFR1) as markers of endometrial unbalance between factors activating the complement system in women with consecutive miscarriages. Study enrolled 30 women with at least two consecutive miscarriages, and 19 healthly women, that comprised the control group. RNA was isolated from endometrial samples. Transcripts levels of DAF and MCP was higher in women with consecutive miscarriages compared to controls, 0.78 vs $5.08(\mathrm{p}<0.001)$ and 0.25 vs 0.17 ( $\mathrm{p}=0.001)$ respectively. In consecutive miscarriages group, DAF and MCP expression was correlated with the C3 expression, with $\mathrm{r}=0.60$; $\mathrm{p}<0.001$ and $\mathrm{r}=0.40 ; \mathrm{p}=0.03$ respectively. Correlation between DAF and $\mathrm{C} 3$ was also noted in controls, $0.70 ; \mathrm{p}=0.001$. In women with two or more consecutive miscarriages the analysis proved higher expression of genes that encode proteins that inhibit the complement cascade. Further studies are needed to confirm that this might be a reaction to increased presence of the complement factors, which like $\mathrm{C} 3$ that are synthesized in the endometrium.
\end{abstract}

Key words: C3, DAF, endometrium, MCP, recurrent miscarriage, the complement system

\section{Introduction}

Apart from classical pathway of the complement activation associated with antibodies, there is also so called alternative pathway for complement system activation. In the alternative pathway the cascade of activation leading to formation of membrane attack complex - MAC and increase in inflammatory reaction could occur spontaneously and is regulated by the action of specific factors. These regulators might be synthesized by leucocytes in the response to C5a complement component, and also when there is insufficient inhibition of complement cascade by certain factors as: decay-accelerating factor (DAF), membrane cofactor protein (MCP), complement receptor 1 -

Correspondence: P. Wirstlein, Dept. of Gynecology and Obstetrics, Division of Reproduction, University of Medical Sciences in Poznań, Polna Str. 33, 60-535 Poznań, Poland; tel.: (+4861) 8419302, fax.: (+4861)8419625, e-mail: abys@wp.pl
CR1. There are synthesized and attached to the surface of attacked cells (in case of immunologic reaction) [1].

In vivo studies in the endometrium have shown increased expression of genes controlling the complement activation during secretory phase of the cycle compared to proliferative phase [2]. In an in vitro studies it was proven that heparin-binding epidermal growth factor-like growth factor (HB-EGF) influences the expression of DAF, MCP in the cells of luminal and glandular endometrium $[3,4]$. Endometrium also synthesizes the $\mathrm{C} 3$ component of the complement system, which is one of the early and key components of the complement system cascade [5]. C3 is a common element for the classic and alternative pathways of the complement activation. It was shown that blocking $\mathrm{C} 3$ gene or protein inhibits the activation of the complement, which had an evident positive effect on decreased resorbtion rates of fetuses in mice model [6].

Explanation of the negative effect of $\mathrm{C} 3$ complement component on embryos, might come from stud- 
Table 1. Clinical characterization of studied groups.

\begin{tabular}{|c|c|c|c|c|c|c|c|}
\hline & \multirow[t]{2}{*}{$\mathrm{n}$} & \multicolumn{2}{|c|}{ Age } & \multicolumn{2}{|c|}{$\begin{array}{c}\text { Number of miscarriages before } 20 \text { week } \\
\text { of gestation }\end{array}$} & \multicolumn{2}{|c|}{ Parity } \\
\hline & & Median & Range & Median & Range & Median & Range \\
\hline Miscarriage group: & 30 & 30 & $22-39$ & 2 & $2-5$ & \multicolumn{2}{|c|}{0} \\
\hline \multirow{2}{*}{$\begin{array}{l}\text { 1. Two consecutive, unexplained } \\
\text { miscarriage } \\
\text { 2. Clinical diagnosed recurrent } \\
\text { miscarriage }\end{array}$} & 22 & 29 & $22-32$ & 2 & 2 & & \\
\hline & 8 & 33 & $28-39$ & 3 & $3-5$ & & \\
\hline Control group & 19 & 39 & $19-47$ & \multicolumn{2}{|c|}{0} & 1 & $1-3$ \\
\hline
\end{tabular}

ies on aPL mice showed that decidual cells, in response to the complement cascade product $\mathrm{C} 5 \mathrm{a}$, release soluble receptor for vascular endothelial growth factor (VEGFR1, syn. SFlt1). Decreasing the availability of vascular endothelial growth factor (VEGF1) for trophoblast VEGF1 receptors leads to decrease in the development of vessels, inhibition or drastic decline in trophoblast invasion and the maldevelopment of the placenta and the fetus [7]. Authors also point to an importance of alternative pathway of the complement activation in enhancement - via a positive feedback loop - of the inflammatory reaction.

The above observations suggest mechanism in which, among others, HB-EGF influences the expression of DAF, MCP. On the other hand, these factors inhibits the complement cascade, of which the C3 component is the key element. Finally, one of the results of complement cascade activation is the sFlt1 synthesis. Problems with inadequate expression of above mentioned factors controlling the complement cascade might be one of the reasons for unexplained, consecutive miscarriages. In current study an attempt was made to compare the expression levels of DAF, MCP, C3, HB-EGF, and VEGFR1 genes. The correlations between $\mathrm{C} 3$ and DAF, MCP transcripts - as direct inhibitors - of complement cascade and correlations between C3 and VEGFR1 - as cascade propagators - transcripts in eutopic endometrium during the implantation window in non-conceptional cycles in women with unexplained, consecutive miscarriage and healthy, fertile women were studied.

\section{Materials and methods}

The study, which obtained acceptance from Karol Marcinkowski's Medical University bioethical committee, included 49 reproductive aged women. The study group consisted of 30 women with two and more unexplained, consecutive miscarriages, and the control comprised of 19 women with normal reproductive potential. The women in a miscarriage group have had at least two consecutive unexplained miscarriages in the first trimester of pregnancy. This group included 8 women with diagnosed recurrent miscarriage(e.g. 3 consecutive miscarriages). The control consisted of women that had at least one child, regular menses, without anatomical or functional changes within the endometrium.
The exclusion criteria were: current use of hormonal contraception, any serious diseases. The age of women, number of miscarriages and parity are showed in Table 1 . In each woman the pipelle or hysteroscopic biopsy was used to acquire the endometrium during the implantation window, which is 7-9 days after ultrasound confirmed ovulation.

RNA Isolation. Part of the biopsy was transferred to histology for evaluation according to Noyes and Hertig criteria [8]; the rest was placed in RNAlater solution (Qiagen, Germany) and frozen to $-80^{\circ} \mathrm{C}$ till RNA isolation. The RNA isolation was conducted with the use of RNAeasy Protect Mini Kit (Qiagen, Germeny). To eliminate the risk of DNA contamination we have used an additional recommended by the manufacturer RNaseFree DNase Set (Qiagen, Germany).

Reverse Transcription. For reverse transcription we taken $1 \mu \mathrm{g}$ previously isolated RNA. Qantity at OD260nm and purity at OD260/280nm samples was checked spectrophotometrically withNanoDrop ND1000 (ThermoScientific, USA). With the use of QuantiTect Reverse Transcription Kit and oligodT (Qiagen, Germany) we have obtained cDNA from previously isolated mRNA fraction. The reverse transcription reaction was antedated by additional elimination of DNA with the use of the QuantiTect Wipeout buffer, one of the components of QuantiTec Reverse Transcription Kit. The matrix that was achieved was free of any contamination from genomic DNA, amplification of which could falsify the real time PCR reaction.

Primers design. Primers for the real time PCR reaction: The glyceraldehyde 3-phosphate dehydrogenase (GAPDH), VEGFR1, MCP primers were constructed based on the RTPrimerDB [9]. Primers for DAF, HB-EGF, beta-actin (ACTB) and C3 were constructed with the use of Primer 3 software [10] and their specificity for cDNA was verified in the BLAST database [11]. Additionally, the thermodynamic results of designed primers were checked against OligoAnalizer software, to confirm the specificity for achieved primers. Primer sequences, size of achieved reaction products and melting temperature (Tm) are depicted in Table 2.

Real Time PCR. All reactions were conducted using DyNAmo HS SYBR Green qPCR Kit (Finnzymes, Finland) and RotorGene 3000 thermocycler (CorbettResearch, Australia). Specificity of achieved reaction products were checked on $2 \%$ agarose gel and second derivative of melting curve for the PCR reaction.

To establish the levels of given transcripts in studied sample we have constructed standard curves generated by the $\mathrm{Ct}$ (value where amplification curve crosses the threshold line ) with seven subsequent 10 times dilutions of linear DNA, which is the product of given set of primers. The quality of achieved curves, described with R2 was contained within 0.9986-0.9992, end the efficiency E 
Table 2. Forward and reverse primer sequences, size of achieved reaction products and melting temperature

\begin{tabular}{|c|c|c|c|c|c|}
\hline \multirow{2}{*}{ Gene } & \multirow{2}{*}{ FF } & \multirow{2}{*}{ REV } & \multirow{2}{*}{$\begin{array}{l}\text { Product } \\
\text { size } \\
\text { (bp) }\end{array}$} & \multicolumn{2}{|c|}{$\operatorname{Tm}\left[{ }^{\circ} \mathrm{C}\right]$} \\
\hline & & & & $\Gamma \mathrm{F}$ & REV \\
\hline HB-EGF & TGAGGTGGGTGGGATTATACA & TTGGAGCTGACTGTTCTTGGT & 93 & 64.2 & 64.2 \\
\hline DAF & TGGTGGTGCTGGACAATAAA & GGTGCAACCATCTCCTTCTC & 141 & 64.3 & 64.0 \\
\hline $\mathrm{MCP}$ & TTGATTGTACCAAGGGATGGA & AACTGCTTGGCTAAGGGACTC & 99 & 64.3 & 63.8 \\
\hline VEGFRl & GGCATGGGAATTGCTTTGG & GACCTGGAGTTACCCTGATGAAA & 76 & 67.7 & 65.5 \\
\hline GAPDH & GGAGTGGGTGTCGCTGTTG & GACGGAAGTGGTTACCTGGA & 109 & 64.2 & 64.5 \\
\hline $\mathrm{ACTB}$ & TGAAGGTAGTTTCGTGGATGC & TCCTCCCTGGAGAAGAGCTCAC & 116 & 64.3 & 64.4 \\
\hline
\end{tabular}

$\mathrm{FF}$ - forward primer; REV - reverse primer; $\mathrm{Tm}$ - melting temperature

Table 3. Transctipts levevs of MCP, HB-EGF, DAF, C3 i VEGFR1 showed as median, and 95CI. The target mRNA levels were corrected to the amount ACTB and GAPDH cDNA and expressed as multiplicity of these cDNA copies in calibrator

\begin{tabular}{|c|c|c|c|c|c|}
\hline & \multicolumn{4}{|c|}{ Gene transcript level } & \multirow{3}{*}{$\mathrm{p}^{*}$} \\
\hline & \multicolumn{2}{|c|}{ Control group $n-19$} & \multicolumn{2}{|c|}{ Unexplained, consecutive miscarriage group $n-30$} & \\
\hline & Median & $95 \mathrm{CI}$ & Median & $95 \mathrm{CI}$ & \\
\hline $\mathrm{MCP}$ & $1.71 \mathrm{E}-01$ & $9.77 \mathrm{E}-02-1.82 \mathrm{E}-01$ & $2.49 \mathrm{E}-01$ & $1.79 \mathrm{E}-01-3.64 \mathrm{~F}-01$ & 0.001 \\
\hline $\mathrm{HB}-\mathrm{EGF}$ & $8.50 \mathrm{E}-03$ & $3.66 \mathrm{E}-03-2.17 \mathrm{E}-02$ & $1.03 \mathrm{E}-02$ & $7.12 \mathrm{E}-03-1.78 \mathrm{E}-02$ & NS \\
\hline $\mathrm{DAF}$ & $5.08 \mathrm{E}-02$ & $8.77 \mathrm{E}-03-3.11 \mathrm{E}-01$ & $7.83 \mathrm{E}-01$ & $3.27 \mathrm{E}-01-1.35 \mathrm{E}+00$ & $<0.001$ \\
\hline $\mathrm{C} 3$ & $4.32 \mathrm{E}-03$ & $9.80 \mathrm{E}-04-7.31 \mathrm{E}-02$ & $4.42 \mathrm{E}-03$ & $1.01 \mathrm{E}-03-1.16 \mathrm{E}-02$ & NS \\
\hline VEGFR1 & $3.82 \mathrm{E}-04$ & $8.20 \mathrm{E}-06-5.00 \mathrm{E}-03$ & 2.37Е-04 & $3.10 \mathrm{E}-07-3.03 \mathrm{E}-03$ & NS \\
\hline
\end{tabular}

*Mann-Whitney Rank Sum Test

of the reaction was within 0.92-1.00. The thermal profile was based on manufacturer's instructions, regarding the specific annealing temperature of primers. The relative expression of studied transcript in given sample was calculated as the ratio of the measured $\mathrm{Ct}$ value to a $\mathrm{Ct}$ value for a reference genes with constitutive expression - GAPDH, ACTB (beta actin). Because the use of different reference genes in different tissues is often a subject of debates we have verified the adequacy of ACTB with GAPDH [12]. The levels of both genes was highly correlated, the correlation coefficients for both genes were 0.915 for the consecutive miscarriage group and 0.841 for the controls $(\mathrm{p}<0.0001)$. The calculation of $\mathrm{Ct}$ values for standards and studied samples and construction of standard curves was performed with the RotorGene 3000 software.

Statistical analysis. For statistical analysis we have used the SigmaStat 3.5 software. For normality of the variables Kolmogorov Smirnov test was used and showed non-parametric distibution of the variables. To compare the relative expression between studied groups we used the Mann-Whitney test. Correlations between studied transcripts were assessed by Spearman analysis and $p<0.05$ was considered statistically significant.

\section{Results}

Achieved results show statistically significant higher relative expression of factors inhibiting the comple- ment activation in women with unexplained consecutive miscarriages. Transcripts levels of MCP was 1.45 and DAF was 15.41 times higher in women with consecutive miscarriages compared to controls. Transcripts levels of HB-EGF, despite being higher in controls, failed to reach statistical significance. There was also no statistically significant difference in relative expression of VEGFR1. Similarly, the C3 component of the complement system exhibited no differences between groups. Table 3 shows the median, and 95\% CI for relative transcripts levels.

The levels of relative expression for $\mathrm{MCP}$ and DAF, when studied with the Spearman test, were positively correlated both in the control and the studied group. Additionally, the expression of MCP and DAF was positively correlated with the $\mathrm{C} 3$ expression in women with two or more unexplained, consecutive miscarriages, and the $\mathrm{C} 3$ component positively correlated with DAF in control, and the observed correlation was stronger in the control. Statistically significant correlations were not observed between $\mathrm{C} 3$ and VEGF1 transcripts levels. The correlation coefficients are depicted in Table 4. 
Table 4. Spearman Rank Correlation between transcripts levels C3 and DAF, MCP, and VEGFR1 in study groups

\begin{tabular}{|c|c|c|c|c|c|c|c|c|c|c|c|}
\hline \multicolumn{10}{|c|}{$\begin{array}{c}\text { Spearman Rank Correlation } \\
\mathrm{n}=19\end{array}$} & \multicolumn{5}{c|}{ Unexplained, consecutive miscarriage group } \\
$\mathrm{n}=30$
\end{tabular}

$\mathrm{NS}$ - statistically not significant

\section{Discussion}

It was the first study in literature which attempted the verification of a working hypothesis, that in endometrium of women with two or more unexplained, consecutive miscarriages there is an elevated synthesis of complement system components, compared to fertile women.

Sugiura-Ogasawara et al. [13] showed that in women with two miscarriages that exhibited higher levels of C3 and C4 in peripheral blood, the next pregnancy also ended in miscarriage. Based on above results and our experience we might speculate that the activation of the complement system occurs in response to the fetal antigens. There are also suggestions that the ability to develop increased complement reaction is a result of the maternal immunological status.

However, as it was proven in mouse model by $\mathrm{Xu}$ et al. [14], the mortality of homozygous fetuses is caused by inability to limit the complement cascade activation. In this process in mice the key role is played by the Crry protein, which has analogous function to human DAF and MCP proteins.

Immunohistologic localization of the human DAF and MCP proteins have shown that they are present not only at the maternal-fetal interface, but also in deciduas, which might point to a fact that also the maternal immune system takes an active part in limiting the complement cascade from developing uncontrolled and damaging immunologic response to the fetus [15].

The results of current paper indicate that the DAF and MCP transcripts level are higher in women with two or more unexplained, consecutive miscarriage compared to controls. This is at clear odds with the assumed hypothesis of the increase the complement cascade in women with unexplained, consecutive miscarriages. It is worth pointing out that in a group of women with two or more unexplained, consecutive miscarriage the transcription level of factors inhibiting the complement activation was correlated with the transcription level of $\mathrm{C} 3$. Concurrently the synthesis of $\mathrm{DAF}, \mathrm{MCP}$ and $\mathrm{C} 3$ proteins in the studied material might suggest that during the implantation window of the non-conceptional cycle the synthesis of pro and anti - activator factors remains in balance. This is further confirmed by the correlation observed between $\mathrm{C} 3$ and DAF, which is responsible for inhibiting the $\mathrm{C} 3$ convertase [1].

The expression of DAF and MCP is stimulated by HB-EGF [3]. In our study the level of transcription for this gene did not differ among groups. It is however impossible at this point, to exclude the role of HBEGF on the expression of factors controlling the complement activation. The path from HB-EGF transcript to an active protein is in this situation dependant on other factors like: protein synthesis, binding to coreceptor-heparin sulfate, the activity of heparanse and steroid hormones $[4,16]$. Earlier works by Krussel et al. [17] proved that in endometrium there is a synthesis of both the soluble and bound to the cell membrane VEGFR1, with its maximal expression observed in midproliferative phase, and minimal at the beginning of the luteal phase. Although the methods used in our paper did not differentiate between the specific forms of the above mentioned receptor, we were able to demonstrate low - in relation to reference - transcript levels of VEGFR1 in midluteal phase of the cycle. The 1.6 times lower expression of VEGFR 1 transcript was accompanied in the group of women with miscarriage by higher levels of DAF and MCP, which indirectly inhibit the secretion of sVEGFR1. However, there was no statistically significant negative correlation between those factors in our material. It should be remembered that comparing just the transcripts levels we acquire only part of the information regarding the gene expression. Spliced mRNA transcripts are only matrix for proteins synthesis, which are often posttranslationally processed (i.e. sVEGFR1). The confirmation of achieved results should expected with protein assays.

To summarise, in women with two or more unexplained, consecutive miscarriage the analysis shows higher transcript levels of genes that encode proteins that inhibit the complement cascade . Further studies are needed to confirm the results of the following study, to precise that this is a reaction to increased presence of the complement factors, which like $\mathrm{C} 3$ are synthesized in the endometrium. 
Acknowledgements: The study was conducted thanks to the European Regional Development Fund and Union for Enterprising People- Competitiveness Program 162/E-392/CD/DFS-4/2004.

\section{References}

[ 1] Gołąb J, Jakóbisiak M, Lasek W, Stokłosa T: Immunologia. Warszawa: Wydawnictwo Naukowe PWN;2008

[2] Lobo SC, Huang ST, Germeyer A, et al: The immune environment in human endometrium during the window of implantation. Am J Reprod Immunol. 2004;52:244-251.

[3] Young SL, Lessey BA, Fritz MA, et al: In vivo and in vitro evidence suggest that HBEGF regulates endometrial expression of human decay-accelerating factor. J Clin Endocrinol Metab. 2002;87:1368-1375.

[4] Lessey BA, Gui Y, Apparao KB, Young SL, Mulholland J: Regulated expression of heparin-binding EGF-like growth factor (HB-EGF) in the human endometrium: a potential paracrine role during implantation. Mol Reprod Dev. 2002; 62:446-455.

[ 5] Sayegh RA, Tao XJ, Awwad JT, Isaacson KB: Localization of the expression of complement component 3 in the human endometrium by in situ hybridization. $J$ Clin Endocrinol Metab. 1996;81:1641-1649.

[ 6] Xu C, D Mao, Holers VM, et al: A critical role for murine complement regulator cry in fetomaternal tolerance. Science. 2000;287:498-501.

[7] Girardi G, Yarilin D, Thurman JM, Holers VM, Salmon JE: Complement activation induces dysregulation of angiogenic factors and causes fetal rejection and growth restriction. J Exp Med. 2006;203:2165-2175.
[ 8] Noyes RW, Hertig AT, Rock J. Dating the endometrial biopsy. Fertil Steril. 1950;1:3-25.

[ 9] http://medgen.ugent.be. Accessed February 10, 2010.

[10] http://fokker.wi.mit.edu/primer3/input.htm. Accessed February 10,2010

[11] http://blast.ncbi.nlm.nih.gov/Blast.cgi. Accessed February 10, 2010

[12] Romanowski T, Markiewicz A, Bednarz N, Bielawski KP: Housekeeping genes as a reference in quantitative real-time RT-PCR. Postepy Hig Med Dosw. 2007;61:500-510

[13] Sugiura-Ogasawara M, Nozawa K, Nakanishi T, Hattori Y, Ozaki Y: Complement as a predictor of further miscarriage in couples with recurrent miscarriages. Hum Reprod. 2006; 21:2711-2714

[14] Xu C, D Mao, Holers VM, et al: A critical role for murine complement regulator cry in fetomaternal tolerance. Science. 2000;287:498-501.

[15] Bulla R, Bossi F, Radillo O, de Seta F, Tedesco F: Placental trophoblast and epitelilal cells as target of maternal immune response. Autoimmunity. 2003;36:11-18.

[16] Kirn-Safran C, D'Souza S, Carson D: Heparan Sulfate Proteoglycans and Their Binding Proteins in Embryo Implantation and Placentation. Semin Cell Dev Biol. 2008;19:187-193

[17] Krussel JS, Casan EM, Raga F, et al: Expression of mRNA for vascular endothelial growth factor transmembraneous receptors Flt1 and KDR, and the soluble receptor sflt in cycling human endometrium. Mol Hum Reprod. 1999;5:452-458.

Submitted: 5 November, 2009 Accepted after reviews: 26 March, 2010 